Results B cells grouped into 4 clusters: GCs (DZ and LZ), plasma cells and memory B cells. DZ, LZ and PC clusters were represented in similar proportions in both conditions; whereas, MemB cells were more expanded in the immunized chimeras. Using the paired single cell BCR sequences and repertoire analysis, we observed clones with clear expansion both in the autoimmune and immunized chimeras. We observed levels of mutation in a similar range though DZ, LZ and MemB from autoimmune mice had a significantly higher number of nucleotide replacement mutations, and the reverse was observed in PCs. Nevertheless, PCs in both conditions reached similar maximum levels of mutation. Interestingly, autoimmune cells showed more isotype diversification in all compartments. Notably, we observed distinct gene expression for the autoreactive B cells such as CXCL10 by GC B cells and SLPI expression by autoreactive plasma cells. Strikingly, we identified DN2- and DN4-like memory B cells in both conditions.

Conclusions We find WT B cells break tolerance, expand in GC and develop into MemB and PCs in a seemingly unrestricted manner, similar to immune mice. Results should open the way to new approaches to control pathogenicity of rogue $\mathrm{B}$ cells in autoimmune disease.

Acknowledgements This work was supported by grants from NIH (R01 AI130307 and AR074105).

\section{ACTIVATED PI3K $\delta$ SIGNALS COMPROMISE PLASMA CELL SURVIVAL VIA LIMITING AUTOPHAGY AND INCREASING ENDOPLASMIC RETICULUM STRESS}

\begin{abstract}
1,2,5 David J Rawlings*, 1,2,3Fahd Al Qureshah, 'Sara Sagadiev, ${ }^{1}$ Christopher D Thouvenel, ${ }^{1}$ Shuozhi Liu, ${ }^{4}$ Zhaolin Hua, ${ }^{4}$ Baidong Hou, ${ }^{1}$ Mridu Acharya, ${ }^{1,5,6}{ }^{1}$ Richard G James. ${ }^{1}$ Seattle Children's Research Institute, Seattle, WA, USA; ${ }^{2}$ Departments of Immunology, University of Washington, Seattle, WA, USA; ${ }^{3}$ King Abdulaziz City for Science and Technology, Riyadh, Saudi Arabia; ${ }^{4}$ Institute of Biophysics, Chinese Academy of Sciences, Beijing, China; ${ }^{5}$ Departments of Pediatrics, University of Washington, Seattle, WA, USA; ${ }^{6}$ Departments of Pharmacology, University of Washington, Seattle, WA, USA
\end{abstract}

\subsection{6/lupus-2021-lupus21 century.98}

Background Understanding key signals that control the differentiation, function, and survival of plasma cells (PCs) is critical for development of improved therapeutic approaches to attenuate pathogenic antibody responses in SLE. While phosphatidylinositide 3-kinase delta (PI3K $\delta$ ) plays an essential role in humoral immune responses, its role(s) in PC function remains poorly understood.

Methods We utilized a conditional mouse model of Activated PI3K $\delta$ Syndrome (APDS), to interrogate the role of this key signaling program.

Results Mice expressing a gain-of-function mutation in PIK3CD in $\mathrm{B}$ cells, referred to as activated (a) PIK3CD, generated increased numbers of memory B cells, mounted enhanced secondary response, yet exhibited a rapid decay of antibody levels over time. Consistent with these findings, aPIK3CD expression markedly impaired plasma cell generation. Remarkably, PC specific aPIK3CD expression was sufficient to diminish humoral responses in vivo. Mechanistically, aPIK3CD disrupted endoplasmic reticulum proteostasis and autophagy, leading to increased PC death. Notably, this defect was driven primarily by elevated mTORC1 signaling and modulated by treatment with $\mathrm{PI} 3 \mathrm{~K} \delta$-specific inhibitors.

Conclusions Taken together, these data demonstrate an unexpected requirement to down-regulate $\mathrm{PI} 3 \mathrm{~K} \delta$ activity to balance autophagy and the unfolded protein response, events essential to modulate ER stress and ensure PC survival. Thus, enhancing $\mathrm{PI} 3 \mathrm{~K} \delta$ activity may provide a novel means to trigger early PC death and dampen autoantibody responses.

\section{IDENTIFYING CLUSTERS OF LONGITUDINAL AUTOANTIBODY PROFILES ASSOCIATED WITH SYSTEMIC LUPUS ERYTHEMATOSUS DISEASE OUTCOMES}

${ }^{1}$ May Y Choi ${ }^{*},{ }^{2}$ Irene Chen, ${ }^{1}$ Ann Clarke, ${ }^{1}$ Marvin J Fritzler, ${ }^{1}$ Katherine A Buhler, ${ }^{3}$ Murray Urowitz, ${ }^{4}$ John G Hanly, ${ }^{5}$ Caroline Gordon, ${ }^{6}$ Yvan St Pierre, ${ }^{7}$ Sang-Cheol Bae, ${ }^{8}$ Juanita Romero Diaz, ${ }^{9}$ Jorge Sanchez-Guerrero, ${ }^{10}$ Sasha Bernatsky, ${ }^{11}$ Daniel Wallace, ${ }^{12}$ David Isenberg, ${ }^{12}$ Anisur Rahman, ${ }^{13}$ Joan T Merrill, ${ }^{14}$ Paul R Fortin, ${ }^{3}$ Dafna D Gladman, ${ }^{15}$ Ian Bruce, ${ }^{16}$ Michelle A Petri, ${ }^{17}$ Ellen Ginzler, ${ }^{18}$ Mary Anne Dooley, ${ }^{19}$ Rosalind RamseyGoldman, ${ }^{20}$ Susan Manzi, ${ }^{21}$ Andreas Jonsen, ${ }^{22}$ Graciela S Alarcon, ${ }^{23}$ Ronald FVan Vollenhoven, ${ }^{24}$ Cynthia Aranow, ${ }^{24}$ Meggan Mackay, ${ }^{25}$ Guillermo Ruiz-Irastorza, ${ }^{26}$ Sam Lim, ${ }^{27}$ Murat Inanc, ${ }^{28}$ Kenneth C Kalunian, ${ }^{29}$ Soren Jacobsen, ${ }^{30}$ Christine Peschken, ${ }^{31}$ Diane Kamen, ${ }^{32}$ Anca Askanase, ${ }^{2}$ David Sontag, ${ }^{33}$ Jill Buyon, ${ }^{34}$ Karen H Costenbader. 'University of Calgary, Cumming School of Medicine, Calgary, Alberta, Canada; ${ }^{2}$ Massachusetts Institute of Technology, Department of Electrical Engineering and Computer Science, Cambridge, MA, USA; ${ }^{3}$ Lupus Program, Centre for Prognosis Studies in The Rheumatic Disease and Krembil Research Institute, Toronto Western Hospital, University of Toronto, Toronto, Ontario, Canada; ${ }^{4}$ Division of Rheumatology, Department of Medicine and Department of Pathology, Queen Elizabeth /I Health Sciences Centre and Dalhousie University, Halifax, Nova Scotia, Canada; ${ }^{5}$ Rheumatology Research Group, Institute of Inflammation and Ageing, College of Medical and Dental Sciences, University of Birmingham, Birmingham, UK; ${ }^{6}$ Research Institute of the McGill University Health Centre; Montreal, Quebec, Canada; 'Department of Rheumatology, Hanyang University Hospital for Rheumatic Diseases, Seoul, Korea; ${ }^{8}$ Instituto Nacional de Ciencias Médicas y Nutrición, Mexico City, Mexico; ${ }^{9}$ Mount Sinai Hospital and University Health Network, University of Toronto, Canada; ${ }^{10}$ Divisions of Rheumatology and Clinical Epidemiology, McGill University Health Centre; ${ }^{11}$ Cedars-Sinai/David Geffen School of Medicine at UCLA, LoS Angeles, California, USA; ${ }^{12}$ Centre for Rheumatology, Department of Medicine, University College London, UK; ${ }^{13}$ Department of Clinical Pharmacology, Oklahoma Medical Research Foundation, Oklahoma City, Oklahoma, USA; ${ }^{14}$ Division of Rheumatology, CHU de Québec - Université Laval, Québec City, Canada; ${ }^{15}$ University of Manchester, Manchester, UK: ${ }^{16}$ Division of Rheumatology, Johns Hopkins University School of Medicine, Baltimore, Maryland, USA; ${ }^{17}$ Department of Medicine, SUNY Downstate Medical Center, Brooklyn, New York, USA; ${ }^{18}$ Thurston Arthritis Research Center, University of North Carolina, Chapel Hill, North Carolina, USA; ${ }^{19}$ Northwestern University and Feinberg School of Medicine, Chicago, Illinois, USA; ${ }^{20}$ Allegheny Health Network, Pittsburgh, Pennsylvania, USA; ${ }^{21}$ Lund University, Lund, Sweden; ${ }^{22}$ Department of Medicine, University of Alabama at Birmingham, Birmingham, Alabama, USA; ${ }^{23}$ University of Amsterdam, Rheumatology and Immunology Center, Amsterdam, Noord-Holland, The Netherlands; ${ }^{24}$ Feinstein Institute for Medical Research, Manhasset, New York, USA; ${ }^{25}$ Autoimmune Diseases Research Unit, Department of Internal Medicine, BioCruces Health Research Institute, Hospital Universitario Cruces, University of the Basque Country, Barakaldo, Spain; ${ }^{26}$ Emory University School of Medicine, Division of Rheumatology, Atlanta, Georgia, USA; ${ }^{27}$ Division of Rheumatology, Department of Internal Medicine, Istanbul Medical Faculty, Istanbul University, Istanbul, Turkey; ${ }^{28}$ University of California San Diego School of Medicine, La Jolla, California, USA; ${ }^{29}$ Department of Rheumatology, Rigshospitalet, Copenhagen University Hospital, Copenhagen, Denmark; ${ }^{30}$ University of Manitoba, Winnipeg, Manitoba, Canada; ${ }^{31}$ Medical University of South Carolina, Charleston, South Carolina, USA; ${ }^{32}$ Hospital for Joint Diseases, New York University, Seligman Centre for Advanced Therapeutics, New York New York, USA; ${ }^{33}$ New York University School of Medicine, New York, NY, USA; ${ }^{34}$ Division of Rheumatology, Inflammation and Immunity, Department of Medicine, Brigham and Women's Hospital, Harvard Medical School, Boston, Massachusetts, USA

\subsection{6/lupus-2021-lupus21 century.99}

Background Prior studies of SLE clusters based on autoantibodies have utilized cross-sectional data from single centers. We applied clustering techniques to longitudinal and comprehensive autoantibody data from a large multinational, multiethnic inception cohort of well characterized SLE patients to identify clusters associated with disease outcomes. 